\title{
Extraordinary transverse magneto-optical Kerr effect in a superlens
}

\author{
E. Moncada-Villa, ${ }^{1,2}$ A. García-Martín, ${ }^{3}$ and J. C. Cuevas ${ }^{2, *}$ \\ ${ }^{1}$ Departamento de Física, Universidad del Valle, AA 25360, Cali, Colombia \\ ${ }^{2}$ Departamento de Física Teórica de la Materia Condensada and Condensed Matter Physics Center (IFIMAC), \\ Universidad Autónoma de Madrid, E-28049 Madrid, Spain \\ ${ }^{3}$ IMM-Instituto de Microelectrónica de Madrid (CNM-CSIC), Isaac Newton 8, PTM, Tres Cantos, E-28760 Madrid, Spain
}

(Received 29 May 2014; revised manuscript received 5 August 2014; published 15 August 2014)

\begin{abstract}
It has been shown that a slab of a negative index material can behave as a superlens enhancing the imaging resolution beyond the wavelength limit. We show here that if such a slab possesses in addition some magnetooptical activity, it could act as an ideal optical filter and exhibit an extraordinary transverse magneto-optical Kerr effect. Moreover, we show that losses, which spoil the imaging resolution of these lenses, are a necessary ingredient to observe this effect.
\end{abstract}

DOI: 10.1103/PhysRevB.90.085120

PACS number(s): 78.20.Ls, 42.25.Gy, 78.20.Ci, 78.66.Bz

As Veselago predicted in his seminal work [1], negative index materials (NIMs) can exhibit very striking electromagnetic properties such as a reversed Doppler effect or negative refraction. This latter property was exploited by Pendry to propose that a slab of a NIM could behave as a superlens [2]; i.e., it could allow the imaging of objects with subwavelength precision. Although the ideal realization of a superlens in the optical regime is quite challenging, the basic ideas behind this concept have been confirmed [3] and Pendry's proposal served as an inspiration that led to the blossom of the field of metamaterials [3-5]. These artificial materials have had an impact in different subfields of optics. Thus for instance, chiral metamaterials, an alternative route to negative refraction, have been shown to boost the magnitude of optical effects such as optical activity, circular dichroism, or Faraday rotation [6-9]. The goal of this work is to show that NIMs and, in particular, superlenses also offer new fascinating possibilities for the field of magneto-optics [10].

In this work we consider a slab of a NIM with magnetooptical (MO) activity inside a host dielectric medium; see Fig. 1. The MO activity can be due to either an external magnetic field or an intrinsic magnetization. We focus here on the analysis of the transverse magneto-optical Kerr effect [10], which consists of the change of the amplitude of reflected $p$-polarized light when the magnetic field or the magnetization is in the plane of the slab but perpendicular to the plane of incidence; see Fig. 1. This effect is often characterized by the following quantity:

$$
\mathrm{TMOKE}=\frac{R_{p p}(+\Pi)-R_{p p}(-\Pi)}{R_{p p}(+\Pi)+R_{p p}(-\Pi)}
$$

which measures the relative change in the reflection probability for $p$-polarized light, $R_{p p}$, upon reversal of the magnetic field or magnetization, $\Pi$. Notice that the maximum of the magnitude of this quantity is equal to 1 . The transversal Kerr effect is the basis of different sensing applications [11] and a great effort is being made to find strategies to enhance this MO effect. Thus, for instance, in the emerging field of magnetoplasmonics researchers combine ferromagnetic

\footnotetext{
*juancarlos.cuevas@uam.es
}

materials with noble metals in a variety of nanostructures to use the excitation of plasmons to enhance the TMOKE [12]. Although a lot of progress has been made in this respect in recent years, experimental TMOKE values are usually well below $10^{-2}$. In this work we show that the magnitude of TMOKE for a superlens with MO activity can reach values very close to 1 . In other words, we demonstrate that this system can behave as an ideal optical filter where the reflected light is suppressed for a given field or magnetization orientation. Moreover, we show that losses, which are so harmful for the imaging capabilities of a superlens, are a necessary ingredient for the occurrence of this extraordinary MO effect.

We consider a slab of thickness $d$ of a material that is embedded in a dielectric medium with a dielectric constant $\varepsilon_{0}$ and a permeability equal to 1 ; see Fig. 1 . The slab material is characterized by a dielectric constant $\varepsilon=\varepsilon^{\prime}+i \varepsilon^{\prime \prime}$ and a permeability $\mu=\mu^{\prime}+i \mu^{\prime \prime}$, which can take arbitrary values. Moreover, we assume that this material has MO activity due to an in-plane magnetic field or magnetization oriented along the $y$ axis; see Fig. 1. The MO activity in this configuration results in an optical anisotropy that can be described by the following dielectric tensor [10]:

$$
\hat{\varepsilon}=\left(\begin{array}{ccc}
\varepsilon & 0 & \varepsilon_{x z} \\
0 & \varepsilon & 0 \\
-\varepsilon_{x z} & 0 & \varepsilon
\end{array}\right),
$$

where $\varepsilon_{x z}=i \alpha m$. Here, $\alpha$ accounts for the magnitude of this off-diagonal element and it is simply proportional to the magnitude of the field or the magnetization, $\Pi$, whereas $m=$ \pm 1 takes into account whether the field or the magnetization is parallel $(m=+1)$ or antiparallel $(m=-1)$ to the $y$ axis. For concreteness, we shall assume here that $\alpha$ is a real constant. Notice also that the permeability remains as a diagonal tensor in our model.

As explained above, our goal is to describe the transverse Kerr effect in this system. This requires computing the reflection probability for $p$-polarized waves for a given angle of incidence, $\theta$, and for both field/magnetization orientations $(m= \pm 1)$. This calculation is relatively straightforward and it can be done with standard techniques of optics in layered media [13]. In our case, we have carried it out within the scattering matrix formalism [14] adapted to anisotropic media 


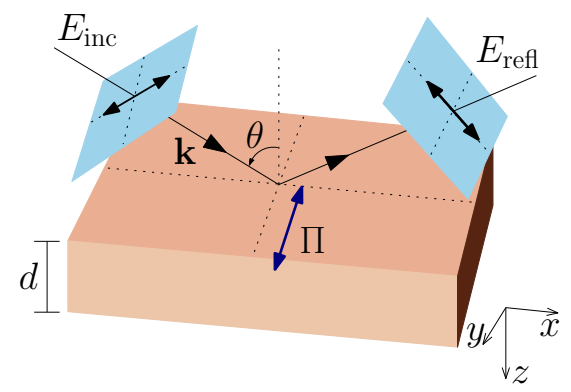

FIG. 1. (Color online) Schematic representation of the transverse magneto-optical Kerr effect in a slab, eventually made of a negative index material, with magneto-optical activity. In this effect a $p$-polarized plane wave impinging on the slab with an angle of incidence $\theta$ is reflected and its amplitude changes depending on the orientation of the external magnetic field or intrinsic magnetization of the slab, $\Pi$, which lies in the plane of the slab and it is perpendicular to the incidence plane.

[15]. The final result for the TMOKE for our configuration is given by

$$
\begin{aligned}
& \text { TMOKE } \\
& =\frac{2 \varepsilon_{0} \alpha\left[\varepsilon_{0} \gamma\left(\varepsilon^{\prime \prime} \mu^{\prime}+\varepsilon^{\prime} \mu^{\prime \prime}\right)-2 \varepsilon^{\prime} \varepsilon^{\prime \prime}\left|\alpha^{2}-\varepsilon^{2}\right|^{2} \cos ^{2} \theta\right] \sin 2 \theta}{\mathcal{D}(\theta)},
\end{aligned}
$$

where

$$
\begin{aligned}
\gamma= & \alpha^{4}+|\varepsilon|^{4}+2 \alpha^{2}\left(\varepsilon^{\prime 2}-\varepsilon^{\prime \prime 2}\right), \\
\mathcal{D}(\theta)= & \left|\alpha^{2}-\varepsilon^{2}\right|^{4} \cos ^{4} \theta-2 \varepsilon_{0}\left|\alpha^{2}-\varepsilon^{2}\right|^{2} \cos ^{2} \theta \\
& \times\left[\varepsilon_{0}\left(\alpha^{2}+\varepsilon^{\prime \prime 2}-\varepsilon^{\prime 2}\right) \sin ^{2} \theta+\varepsilon^{\prime} \mu^{\prime}\left(|\varepsilon|^{2}-\alpha^{2}\right)\right. \\
& \left.+\varepsilon^{\prime \prime} \mu^{\prime \prime}\left(|\varepsilon|^{2}+\alpha^{2}\right)\right]+\varepsilon_{0}^{2} \gamma\left[\varepsilon_{0}^{2} \sin ^{4} \theta+\alpha^{2} \sin ^{2} 2 \theta\right. \\
& \left.-2 \varepsilon_{0}\left(\varepsilon^{\prime} \mu^{\prime}-\varepsilon^{\prime \prime} \mu^{\prime \prime}\right) \sin ^{2} \theta+|\mu \varepsilon|^{2}\right] .
\end{aligned}
$$

Before addressing the case of a superlens, it is worth remarking on two general conclusions that can be drawn from Eq. (3). First, notice that TMOKE is independent of the thickness of the slab, although the reflection coefficients $R_{p p}( \pm \Pi)$ are not. This is due to the fact that we are considering a symmetric system where the medium of incidence and the substrate are made of the same material. More importantly, Eq. (3) tell us that TMOKE vanishes if there are no losses in the slab $\left(\varepsilon^{\prime \prime}, \mu^{\prime \prime}=0\right)$. On the other hand, let us also be reminded that to observe a finite TMOKE one needs to have oblique incidence, and due to the factor $\sin 2 \theta$ in the numerator of Eq. (3), TMOKE reaches its maximum close to $45^{\circ}$ in conventional structures.

Let us now consider the case of a slab with $\varepsilon^{\prime}=-1$ and $\mu=-1$ surrounded by air $\left(\varepsilon_{0}=1\right)$. In the absence of MO activity, this corresponds to the ideal superlens considered by Pendry [2], where the transmission is equal to 1 , irrespective of the angle of incidence. In Fig. 2(a) we show the TMOKE as a function of the angle of incidence for a case where $\alpha=0.05$ and $\varepsilon^{\prime \prime}=0.05$. As one can see, the magnitude of TMOKE reaches the value of 1 at two different angles. More importantly, as we show in Fig. 2(b) these maxima are associated with the blocking of the reflection for one of the field or magnetization orientations. Thus, we see that a superlens
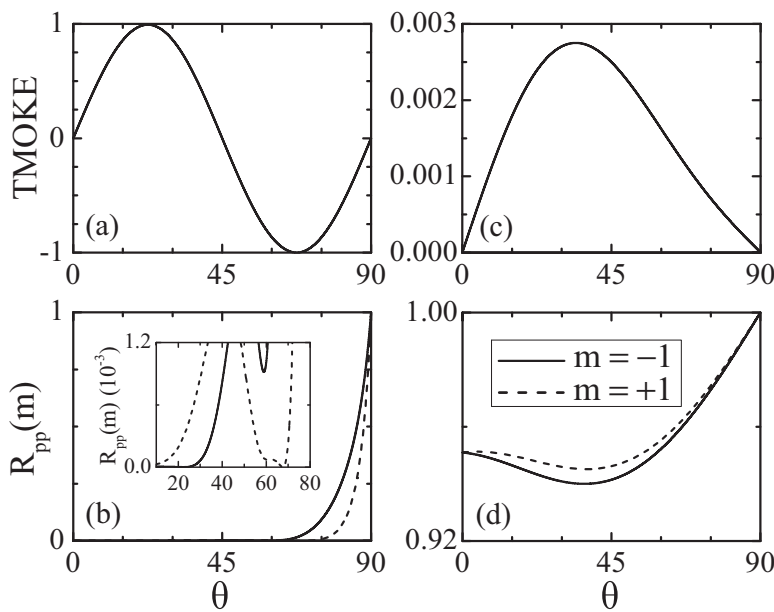

FIG. 2. (a) TMOKE as a function of the angle of incidence for a slab with $\varepsilon=-1+0.05 i, \mu=-1$, and $\alpha=0.05$, surrounded by air. (b) Reflection probabilities for $p$-polarized light corresponding to the results of panel (a) for the two orientations of the field or magnetization. The inset shows a blow-up where one can see the suppression of these probabilities at certain angles. (c)-(d) The same as in panels (a) and (b), but for a permeability equal to +1 . In all cases the slab thickness, $d$, was chosen to be equal to the wavelength. While TMOKE does not depend on $d$, the reflection probabilities do.

with MO activity can behave as an optical filter exhibiting the highest TMOKE possible. Notice that the values of $R_{p p}$ are small for both orientations because we are close to the condition for a perfect lens. However, these values can be tuned to some extent by changing the slab thickness without modifying the TMOKE. For comparison, we show in Figs. 2(c) and 2(d) the corresponding results for a slab where the sign of the permeability has been reversed, $\mu=+1$. In this case, the TMOKE has conventional values on the order of $10^{-3}$ and there is no filtering effect.

Now, in order to illustrate the role of the losses in this extraordinary TMOKE, we show in Fig. 3(a) TMOKE as a function of $\theta$ for a slab with negative index of refraction, but different values of the imaginary part of the dielectric constant, $\varepsilon^{\prime \prime}$, while assuming that $\mu$ is real $\left(\mu^{\prime \prime}=0\right)$. As one can see, the role of the value of $\varepsilon^{\prime \prime}$ is to determine the position of the maxima of |TMOKE|, which still reach the value of 1 . Notice that for small values of $\varepsilon^{\prime \prime}$, those maxima appear close to $0^{\circ}$ and $90^{\circ}$, while they are shifted progressively toward $45^{\circ}$ as the losses increase. Again, this behavior differs dramatically from the one observed when $\mu=+1$; see Fig. 3(b). For the superlens case, the position of the maxima $|\mathrm{TMOKE}| \approx 1$ can be obtained with the help of Eq. (3). When the surrounding medium is air, the positions of the maximum and minimum of TMOKE for $\varepsilon=-1+i \varepsilon^{\prime \prime}$ and $\mu^{\prime \prime}=0$ are given by

$$
\tan 2 \theta=-\frac{m \varepsilon^{\prime \prime}}{\alpha},
$$

where $m=-1$ corresponds to the maximum and $m=+1$ to the minimum of TMOKE, respectively. Thus, we see that those positions are simply governed by the ratio between $\varepsilon^{\prime \prime}$ and $\alpha$, the constant that determines the MO activity of the slab. 

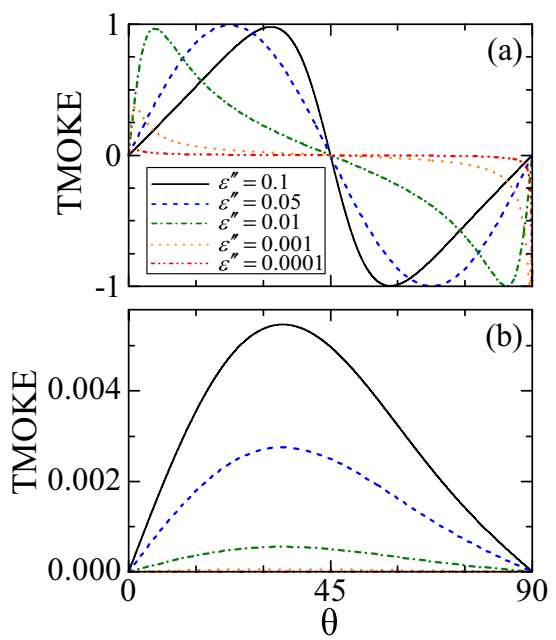

FIG. 3. (Color online) Dependence of the TMOKE with $\varepsilon^{\prime \prime}$ for (a) a superlens $\left(\varepsilon=-1+i \varepsilon^{\prime \prime}\right.$ and $\left.\mu=-1\right)$ and (b) a metallic slab ( $\varepsilon=-1+i \varepsilon^{\prime \prime}$ and $\mu=1$ ). In both cases, $\alpha=0.05$. Notice that these results are independent of the slab thickness.

In general, both the permittivity and the permeability can have real and imaginary parts $[16,17]$. For this reason, it is interesting to briefly discuss the role of the imaginary part of the permeability, $\mu^{\prime \prime}$. In Fig. 4(a) we show the TMOKE as a function of $\theta$ for the case of Fig. 2(a), where $\varepsilon=-1+0.05 i$ and $\mu^{\prime}=-1$, for different values of $\mu^{\prime \prime}$. As one can see, a finite value of $\mu^{\prime \prime}$ asymmetrizes the angular dependence of the TMOKE, but its magnitude can still reach values very close to 1. On the other hand, Fig. 4(b) displays the influence of $\mu^{\prime \prime}$ on the angular dependence of the TMOKE for a case where the permittivity has no imaginary part $(\varepsilon=-1)$. In this case, two features are worth remarking. First, if $\mu^{\prime \prime}=0$ the TMOKE vanishes, which is evident from Eq. (3) and confirms
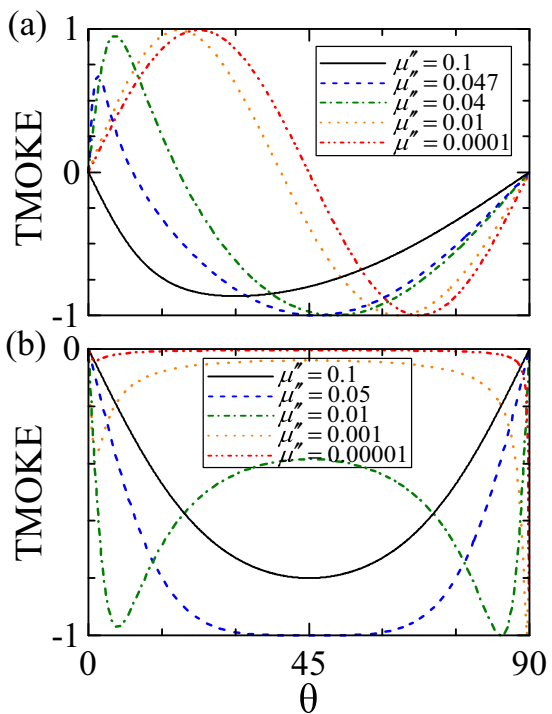

FIG. 4. (Color online) (a) TMOKE as a function of the angle of incidence for a slab with $\varepsilon=-1+0.05 i, \alpha=0.05$, and $\mu=$ $-1+i \mu^{\prime \prime}$. The different curves correspond to different values of $\mu^{\prime \prime}$. (b) The same as in panel (a), but for $\varepsilon=-1$. that losses are necessary to observe this MO effect. Second, for finite $\mu^{\prime \prime}$ the magnitude of the TMOKE can reach again values very close 1 for certain angles of incidence. Thus, we see that the extraordinary TMOKE may appear for a broad range of values of $\varepsilon^{\prime \prime}$ and $\mu^{\prime \prime}$ by simply tuning the angle of incidence.

To gain some further insight into the origin of the extraordinary TMOKE of a superlens, we have analyzed the field distribution inside the slab. In Fig. 5 we show these distributions for the cases shown in Fig. 2 for those angles at which a maximum or a minimum of the TMOKE appears. The figure reflects that the effect of the MO element in the electromagnetic field profile is substantially larger for the superlens case than for the conventional metal. In the normal-metal case, we can see that the field experiences the expected exponential decay inside the metallic layer, with a small perturbation due to the MO element. In the superlens case the decay within the slab is no longer exponential, but much slower. In fact, one can see that the field present some oscillations, except for one of the orientations of the magnetic field. Interestingly, it is the "filtered" component, i.e., the one that is not reflected, the one with damped oscillations, exhibiting an almost linear decay for the three components of the electromagnetic field inside the slab.

As is evident from Eq. (3), the TMOKE is sensitive to the composition of surrounding media. This effect is illustrated in Fig. 6(a), where we show TMOKE as a function of both $\theta$ and $\varepsilon_{0}$, the dielectric constant of the surrounding medium, for $\varepsilon^{\prime}=-2$ and $\mu=-1$. Notice that when $\varepsilon_{0}>\varepsilon^{\prime}$ the maximum of TMOKE disappears, that is, the reflection coefficient $R_{p p}$ is more suppressed at a certain angle when the field or the magnetization is parallel to the $y$ axis. However, when $\varepsilon_{0}<\varepsilon^{\prime}$ only the maximum survives. Notice also that the pattern discussed above with a maximum close to 1 and a minimum close to -1 is recovered when the superlens condition $\varepsilon^{\prime}=-\varepsilon_{0}$ is satisfied. This is illustrated in Fig. 6(b) where we show that if this condition is fulfilled, one recovers the extraordinary MO effect discussed above. What is so special about this condition and, more generally, what does determine the extrema of the TMOKE? It turns out that these extrema are linked to the existence of surface plasmon polaritons (SPPs) in the interface between the slab and the dielectric. To prove this idea, we can make use of the fact that the TMOKE in our symmetric arrangement is independent of the slab thickness and consider a semi-infinite slab. In this case, the SPPs supported by the interface between the slab and the dielectric are given by the condition

$$
\eta_{0} q_{0}+\eta_{x x} q-\eta_{x z} k_{\|}=0 .
$$

Here, $k_{\|}=(\omega / c) \sqrt{\varepsilon_{0}} \sin \theta$ is the SPP wave vector component parallel to the interface and $\omega$ is the radiation frequency. On the other hand, $q_{0}$ and $q$ are the transversal components of the wave vector in the dielectric and in the slab, respectively, and they are given by $q_{0}^{2}=\varepsilon_{0} \omega^{2} / c^{2}-k_{\|}^{2}$ and $q^{2}=\varepsilon \mu \omega^{2} / c^{2}-k_{\|}^{2}$. Finally, $\eta_{0}=1 / \varepsilon_{0}, \eta_{x x}=\varepsilon /\left(\varepsilon^{2}+\varepsilon_{x z}^{2}\right)$, and $\eta_{x z}=-\varepsilon_{x z} /\left(\varepsilon^{2}+\varepsilon_{x z}^{2}\right)$. Equation (5) can be solved to obtain the angle of incidence that corresponds to the existence of the SPPs for both orientations of the magnetic field or the magnetization $(m= \pm 1)$. In Figs. 6(a) and 6(b) we show that the solution of Eq. (5) nicely 


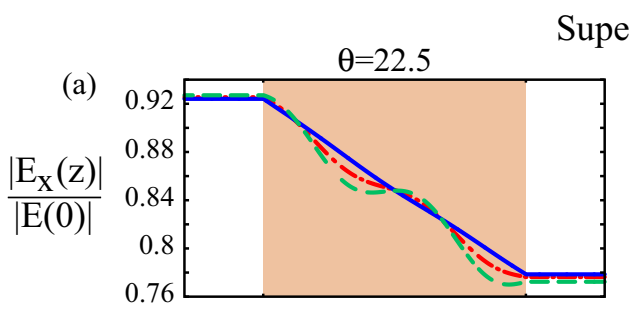

Superlens
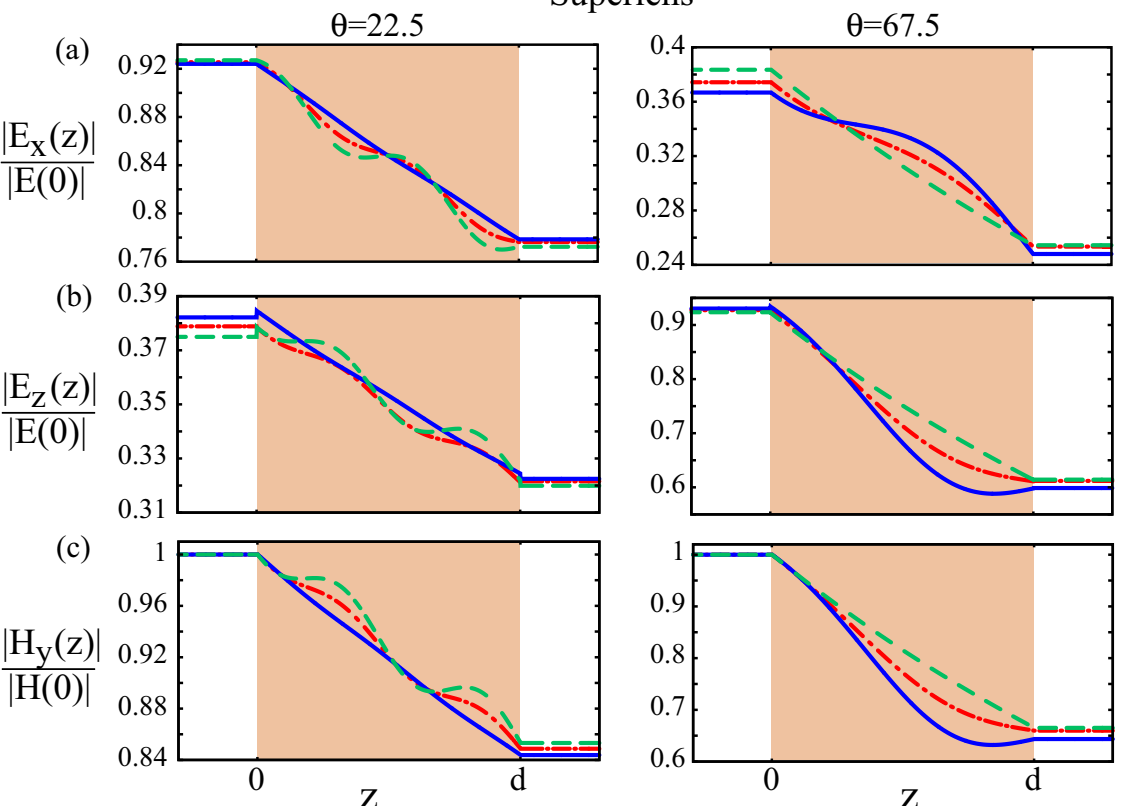
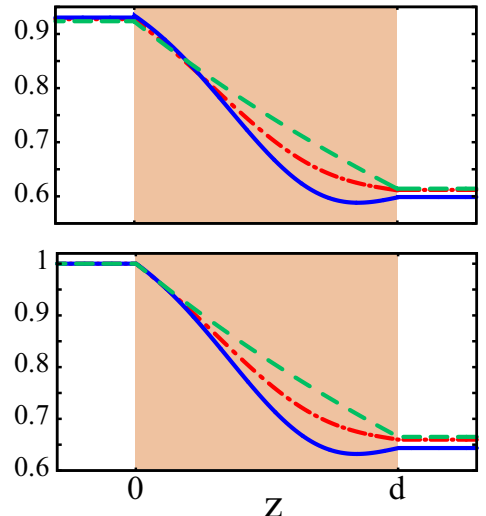

(d)

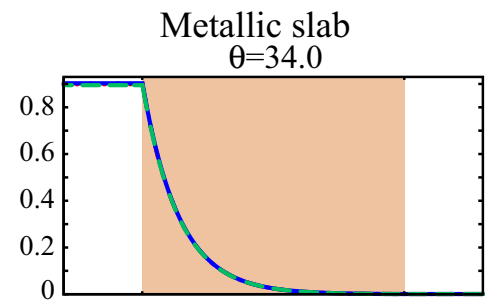

(e)

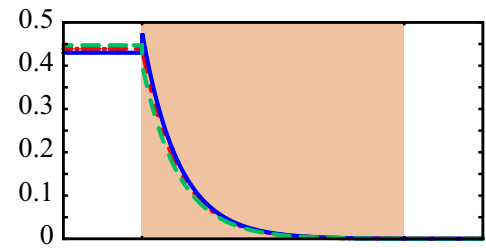

(f)

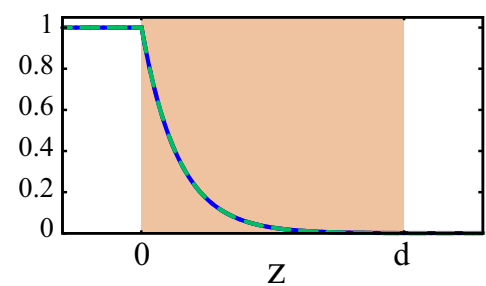

FIG. 5. (Color online) Spatial profiles of nonvanishing field components of a $p$-polarized wave propagating through a superlens, panels (a)-(c), and a metallic slab, panels (d)-(f). These field distributions correspond to the maximum (minimum) of TMOKE at $\theta=22.5(\theta=67.5)$ in Fig. 2(a) for the superlens, and to the maximum occurring at $\theta=34$ in Fig. 2(c) for the metallic slab. Dashed and solid curves correspond, respectively, to a magnetized slab with magnetization parallel $(m=1)$ and antiparallel $(m=-1)$ to the $y$ axis, and the dash-dotted curves to the unmagnetized slab $(\alpha=0)$.

reproduces the position of the extrema of the TMOKE for the whole range of parameters. Thus, the physical picture that emerges is that at certain angles the reflectivity for a given orientation of the magnetization or the magnetic field is partially suppressed due to the excitation of a SPP, which leads to a maximum or a minimum in the TMOKE. In particular, close to the superlens condition $\left(\varepsilon^{\prime}=-\varepsilon_{0}\right)$, the SPPs appear at very different angles of incidence for the two orientations; see Fig. 6(a). Therefore, the extraordinary TMOKE (with a magnitude close to 1) is due to the fact that the reflectivity is largely suppressed only for one orientation of the magnetic

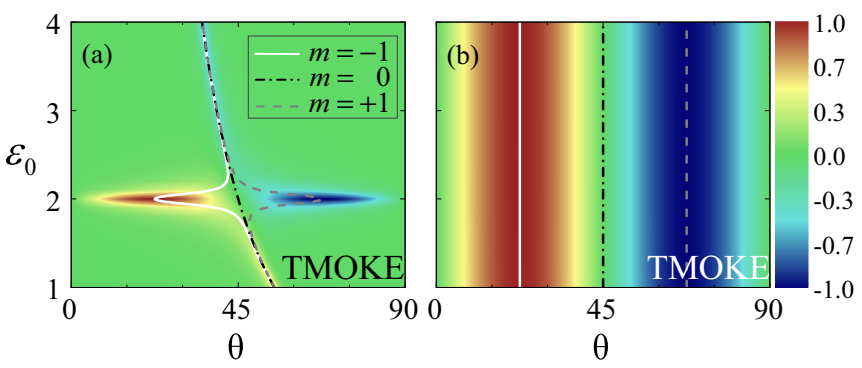

FIG. 6. (Color online) (a) TMOKE as a function of incidence angle and the dielectric constant $\varepsilon_{0}$ of the surrounding medium when $\varepsilon^{\prime}=-2$. The other parameters of the slab are of in Fig. 2(a). (b) The same as in panel (a), but assuming that $\varepsilon^{\prime}=-\varepsilon_{0}$. In both panels the solid and dashed lines correspond to the condition of the existence of surface plasmon polaritons, as given by Eq. (5), for the two possible orientations of the magnetization or the magnetic field $(m= \pm 1)$, while the dotted-dashed corresponds to the condition without MO activity $(\alpha=0)$. field or the magnetization. It is important to stress that the SPPs described here have parallel wave vectors that lie on the left-hand side of the light lines of both the dielectric and the slab. Thus, these modes are not really confined to the interface and they can be excited by a plane wave in the situation described here.

Let us now briefly discuss possible realizations of a superlens with MO activity. In the microwave regime, it has been demonstrated, for instance, that metals can be combined with insulated ferrites, such as magnetized yttrium iron garnet (YIG), to fabricate metamaterials with a tunable negative index of refraction [18]. Naturally, these hybrid ferromagnetic structures must also exhibit some degree of MO activity. Related to this, it has been theoretically suggested that magnetophotonic crystals made of ferromagnetic nanowires can be good candidates for NIMs and they could exhibit an anomalous Faraday effect in virtue of their negative index [19]. On the other hand, although the realization of a superlens in the optical regime has turned out to be quite challenging, there have been relatively successful strategies based, for instance, on two-dimensional metal-insulator-metal (MIM) plasmonic slab waveguides [20]. Moreover, it has been suggested that the practical limitations of these planar MIM geometries can be circumvented by using coaxial MIM waveguides embedded in a plasmonic crystal [21]. Then, the use of ferromagnetic garnets as insulators in these structures might give rise to systems with a simultaneous negative index of refraction and MO activity in the optical regime.

So in summary, we have shown that a superlens made of a slab of a NIM with MO activity could exhibit an extraordinary 
transverse magneto-optical Kerr effect. In particular, we have shown that irrespective of the slab thickness such a system could act as an ideal optical filter where losses are necessary, rather than harmful. We have attributed this very peculiar effect to the existence of surface plasmon polaritons in the interface between the dielectric and the slab. Our work illustrates that NIMs may also have a strong impact in the field of magneto-optics and we believe that our work may trigger more theoretical and experimental investigations of this interesting interplay.

[1] V. G. Veselago, Sov. Phys. Usp. 10, 509 (1968).

[2] J. B. Pendry, Phys. Rev. Lett. 85, 3966 (2000).

[3] V. M. Shalaev, Nat. Photonics 1, 41 (2007).

[4] W. Cai and V. M. Shalaev, Optical Metamaterials: Fundamentals and Applications (Springer, New York, 2009).

[5] O. Hess, J. B. Pendry, S. A. Maier, R. F. Oulton, J. M. Hamm, and K. L. Tsakmakidis, Nature Mater. 11, 573 (2012).

[6] T. G. Mackay and A. Lakhtakia, Phys. Rev. E 69, 026602 (2004).

[7] C. Zhang and T. J. Cui, Appl. Phys. Lett. 91, 194101 (2007).

[8] W. Zhu, I. D. Rukhlenko, F. Xiao, and M. Premaratne, J. Appl. Phys. 115, 143101 (2014).

[9] For a review, see B. Wang, J. Zhou, T. Koschny, M. Kafesaki, and C. M. Souloulis, J. Opt. A: Pure Appl. Opt. 11, 114003 (2009).

[10] A. Zvezdin and V. Kotov, Modern Magnetooptics and Magnetooptical Materials (IOP, Bristol, 1997).

[11] B. Sepúlveda, A. Calle, L. M. Lechuga, and G. Armelles, Opt. Lett. 31, 1085 (2006).
We thank M. Nieto-Vesperinas and J. J. Sáenz for enlightening discussions. E.M.-V. was financially supported by the Colombian agency COLCIENCIAS. A.G.-M. acknowledges funding from Spanish Ministry of Economy and Competitiveness through grants "FUNCOAT" CONSOLIDER CSD2008-00023 and "MAPS" MAT2011-29194-C02-01, and from Comunidad de Madrid through grant "MICROSERESCM" S2009/TIC-1476. J.C.C. acknowledges financial support from the Spanish Ministry of Economy and Competitiveness (Contract No. FIS2011-28851-C02-01).

[12] G. Armelles, A. Cebollada, A. García-Martín, and M. U. González, Adv. Optical Mater. 1, 10 (2013).

[13] P. Yeh, Optical Waves in Layered Media (John Wiley \& Sons, New York, 1988).

[14] D. M. Whittaker and I. S. Culshaw, Phys. Rev. B 60, 2610 (1999).

[15] B. Caballero, A. García-Martín, and J. C. Cuevas, Phys. Rev. B 85, 245103 (2012).

[16] X. Xiong, W.-H. Sun, Y.-J. Bao, M. Wang, R.-W. Peng, C. Sun, X. Lu, J. Shao, Z.-F. Li, and N.-B. Ming, Phys. Rev. B 81, 075119 (2010).

[17] Y. Liu and X. Zhang, Chem. Soc. Rev. 40, 2494 (2011).

[18] H. Zhao, J. Zhou, L. Kang, and Q. Zhao, Opt. Express 17, 13373 (2009).

[19] H. X. Da, C. Xu, and Z. Y. Li, Eur. Phys. J. B 45, 347 (2005).

[20] H. J. Lezec, J. A. Dionne, and H. A. Atwater, Science 316, 430 (2007).

[21] S. P. Burgos, R. de Waele, A. Polman, and H. A. Atwater, Nature Mater. 9, 407 (2010). 